\title{
Status of fission fragment observables measured with the LOHENGRIN spectrometer
}

\author{
S. Julien-Laferrière ${ }^{1,2}$, L. Thombansen ${ }^{l}$, G. Kessedjian ${ }^{l}, A$. Chebboubi ${ }^{2}$, O. Serot $^{2}, C$.

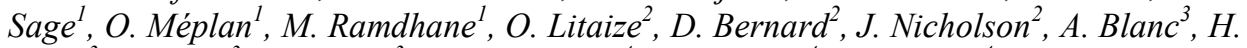

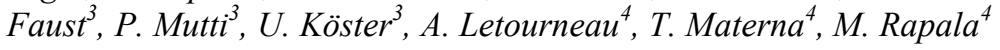 \\ ${ }^{1}$ LPSC, Université Grenoble-Alpes, CNRS/IN2P3, F-38026 Grenoble Cedex, France \\ ${ }^{2}$ CEA, DEN, DER, SPRC, LEPh, Cadarache center, F-13108 Saint Paul lez Durance, France \\ ${ }^{3}$ Institut Laue-Langevin, F-38042 Grenoble Cedex 9, France \\ ${ }^{4}$ CEA, DSM, IRFU, SPhN, LEARN, Saclay center, F-91191 Gif-sur-Yvette, France
}

\begin{abstract}
Nuclear fission yields are key parameters to evaluate reactor physics observables, such as fuel inventory, decay heat, spent fuel radiotoxicity, criticality but also for understanding the fission process. Despite a significant effort allocated to measure fission yields during the last decades, the recent evaluated libraries still need improvements in particular in the description of the uncertainties with the associated correlations. Additional kinds of measurements provide complementary information in order to test the models used in the nuclear data evaluation. Moreover, some discrepancies between these libraries must be explained. A common effort by the CEA, the LPSC and the ILL aims at tackling these issues by providing precise measurement of isotopic and isobaric fission yields with the related variance-covariance matrices. Nevertheless, the experimental program represents itself a large range of observables requested by the evaluations: isotopic yields, nuclear charge polarization, odd-even effect, isomeric ratio and their dependency with fission fragment kinetic energy as a probe of the nuclear de-excitation path in the $\left(E^{*}, J^{\pi}\right)$ representation. Measurements for thermal neutron induced fission of ${ }^{241} \mathrm{Pu}$ have been carried out at the Institut Laue Langevin using the LOHENGRIN mass spectrometer. Experimental program, observables reachable, results and comparison to model calculations are shown.
\end{abstract}

\section{Fission studies for applications}

The collaboration is involved in an experimental program using thermal neutrons to study the fission process and to answer the specific requests concerning the fission yields for the current and innovative fuel cycle studies. Innovative cycles cover the multirecycling of the plutonium in the GEN-III reactors or the studies involving the thorium cycle. Nevertheless, for the actual generation of reactors, improvements on the knowledge of fission yields are expected for different goals:

- Fission fragments as probes of the fuel burn-up or the neutron fluency using cumulative yields (e.g. $\left.{ }^{140} \mathrm{Ba},{ }^{147} \mathrm{Nd}\right)$; 
- Fission yields are input data for the fuel inventory, the decay heat calculations and the Beta/neutrino spectra calculations (using independent yields associated with reaction and decay data);

- Probes of impact of the dynamics in the fission process, in particular the descent from the saddle point to the scission point;

- Fission yields are used for the determination of the prompt particles spectra emitted by the fission fragments. For instance, the calculation of the total prompt gamma spectra for all actinides present in the fuel are required for the determination of the reactor vessel heating and is relying on models calculations.

The program on fission yields with the LOHENGRIN spectrometer at ILL has been initiated for several years [1-2] and we developed different methodologies to obtain absolute fission yields with the description of the Probability Density Function (PDF) and the covariance matrices associated to the measurements [3]. This information on measurements is required in order to consider statistical tests as the cornerstone of the evaluation program [4-5].

\section{Experimental setup}

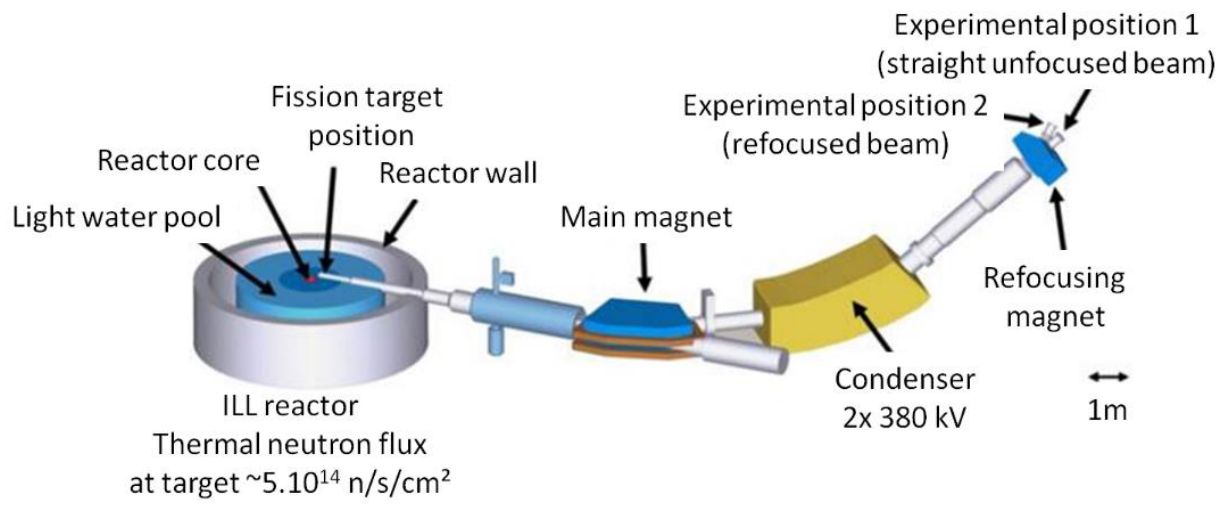

Fig. 1. The LOHENGRIN mass spectrometer at the ILL, Grenoble. The ILL high neutron flux compensates the small geometrical acceptance of the instrument requested for the mass resolution.

The LOHENGRIN recoil mass spectrometer $[6,7]$ of which a descriptive sketch is shown on Fig. 1, has been built in the 70's at the ILL and is since a major instrument for nuclear data measurements in particular due to its high mass resolution $(\Delta \mathrm{A} / \mathrm{A} \sim 1 / 400)$. A purify ${ }^{241} \mathrm{Pu}$ target (see Table 1) is placed close to the reactor core under a high thermal neutron flux $\left(\sim 5 \cdot 10^{14} \mathrm{n} / \mathrm{s} / \mathrm{cm} 2\right.$ at target position) and undergoes a significant amount of fission fragments from ${ }^{241} \mathrm{Pu}(\mathrm{n}, \mathrm{f})$ (more than $98.85 \%$ during 15 days irradiation). Fission products which enter in the apparatus (solid angle $\leq 3.2 \cdot 10^{-5} \mathrm{sr}$ ) are deflected through a magnetic and an electric fields. The ion beam is selected according to the $\mathrm{A} / \mathrm{q}$ and $\mathrm{E}_{\mathrm{k}} / \mathrm{q}$ ratios, where $\mathrm{A}$ is the fission product mass, $\mathrm{E}_{\mathrm{k}}$ its kinetic energy and $\mathrm{q}$ its ionic charge. The ratios $\mathrm{A} / \mathrm{q}$ and $E_{k} / q$ can be achieved with different triplet $\left(A, E_{k}, q\right)$ leading to a possible degeneracy. Two experimental positions exist, at position 1, the ion beam directly enters in an ionization chamber. This position is used to measure isobaric yields, $\mathrm{Y}(\mathrm{A})$ with a dual anode Frisch grid ionization chamber. At position 2, the ion beam is refocused and implanted on a 
moving tape. Two clovers of four high purity germanium crystals each are placed in the vicinity of the tape and detect $\gamma$-rays resulting from the fission products $\beta$ - disintegration, allowing to measure isotopic yields $\mathrm{Y}(\mathrm{A}, \mathrm{Z})$.

Table 1. Initial target composition and limits on fission rates after 6 day irradiation for the July 2016 experiment with the LOHENGRIN spectrometer.

\begin{tabular}{|c|c|c|}
\hline Actinide & Composition (\%) & $\begin{array}{c}\text { Relative } \\
\text { (n,f) rates } \\
(\%)\end{array}$ \\
\hline${ }^{238} \mathrm{Pu}$ & $<0.0003$ & $<110^{-3}$ \\
\hline${ }^{239} \mathrm{Pu}$ & 0.02 & $<3.110^{-2}$ \\
\hline${ }^{240} \mathrm{Pu}$ & 5.30 & $<710^{-4}$ \\
\hline${ }^{241} \mathrm{Pu}$ & 71.20 & $>98.85$ \\
\hline${ }^{242} \mathrm{Pu}$ & 17.40 & $<1.510^{-2}$ \\
\hline${ }^{241} \mathrm{Am}$ & 6.06 & $<0.0410^{-2}$ \\
\hline${ }^{242 \mathrm{GS}} \mathrm{Am}$ \\
$+{ }^{242 \mathrm{~m}} \mathrm{Am}$ & - & $<1.039$ \\
\hline
\end{tabular}

\section{Experimental program: isotopic and isomeric yields}

The first goal of the experiments consists in the precise measurements of mass, isotopic and isomeric yields with a control of the systematic effects and the determination of the covariance matrices associated to the analyses. For these observables, their dependency with fission fragment kinetic energy increases substantially the information on fission process. Theoretically, the full characterization of fission events is provided by the determination of the yield as a function of the mass $A$, the nuclear charge $Z$, the excitation energy $E^{*}$ and the spin-parity $\left(J^{\pi}\right)$ for each fragment: $Y\left(A, Z, E^{*}, J^{\pi}\right)$. Unfortunately, this quantity cannot be determined experimentally. The experimental observables correspond to the fission yields as a function of the kinetic energy $E_{k}$ and the ratio of the isomeric over ground states populations (IR): Y(A, Z, $E_{k}$, IR). Usually the kinetic energy dependency of these populations is described using ratio measurements of isotopes or isomers in order to limit the systematic corrections and to increase the precision (1-5\% achievable). However, the interpretation of these ratios is not straightforward. Indeed, the comparison of experimental and theoretical IR implies the use of several models with different degree of impact. For instance, the sharing of the excitation energy between both fragments (for instance [8-10]) as well as the mechanism by which these fragments acquire their angular momenta, the level density models, the gamma strength function and the spin parity distribution before and after prompt neutron emission [11] must be considered. Thus, isotopic and isomeric ratios are the crucial observables to assess the dynamical aspects of the fission process.

In references [12] [13], the authors described two experiments performed at LOHENGRIN to illustrate the expected dependence of the isomeric ratio as a function of the kinetic 
energy. Different experimental methodologies are being developed today by the collaboration to measure and interpret isotopic and isomeric yields. The goal is the construction of a library of measurements in order to test the decay cascade on a large range of isotopes.

\subsection{Isotopic yields and charge polarization}

Isotopic and long-life isomeric (few $\mu$ s to ms) yields are now currently measured with the LOHENGRIN spectrometer using the gamma spectrometry. This consists in a cumulative measurement with duration of about 30 min per point over the kinetic energy or ionic charge distributions. After corrections for the radioactive decays of the fragments, we obtain the isotopic yields (see Fig. 2). Particular effort is oriented on the determination of the systematic uncertainties and the covariance associated to the measurement process [3]. From this data, combined with mass yields measurements, we are able to extract the nuclear charge polarization (see Fig. 3). If we observed a good agreement with the JEFF3.1.1 and JEFF3.3 libraries for the mass 130 and around mass 140, a strong structure appears for the masses 132, 136 and 138. This region corresponds to the already observed structures at $\mathrm{Z}=50$ and $\mathrm{Z}=54$. Complementary measurements on the neighboring masses are planned at ILL to better understand this phenomenon.
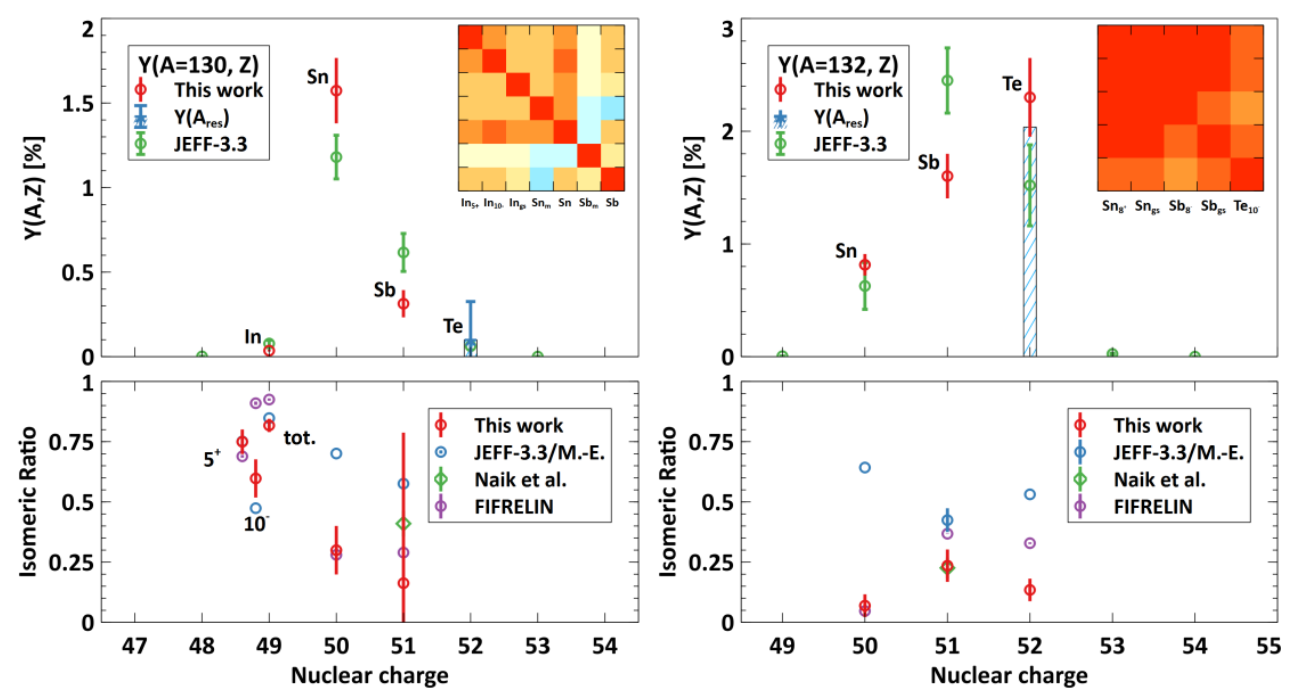

Fig. 2. Results for the masses 130 (left) and 132 (right). (Top plots) Isotopic yields with their correlations have been compared with the library JEFF-3.3. The hatched bars correspond to the residual isotopic yields which are not measured with gamma spectrometry but deduced from the mass yields measurements. (Bottom plots) Isomeric ratios for different isotopes compared to the FIFRELIN calculations, the JEFF-3.3 evaluations and the H. Naik et al. interpretations [14]. 


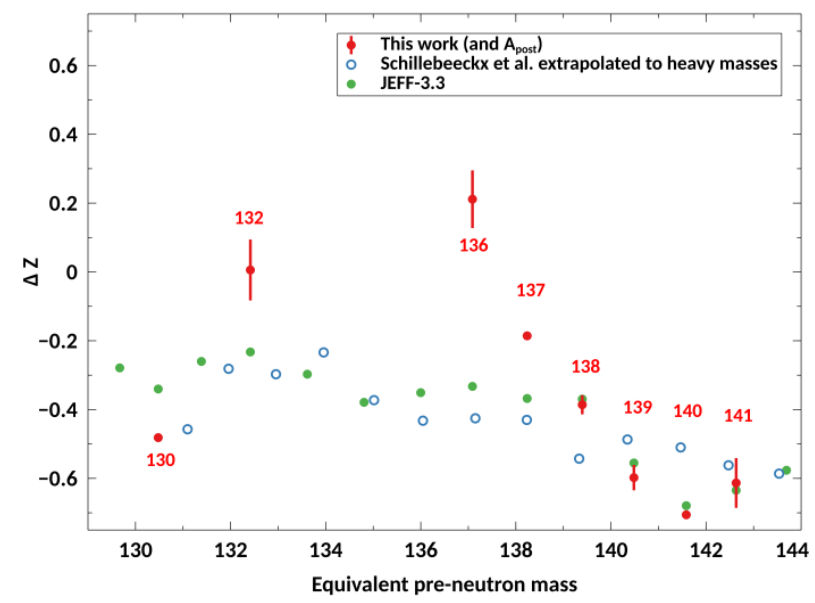

Fig. 3: Our measurements (red points labeled with the measured post-neutron emission mass $\left.A_{\text {post }}\right)$ ) of the nuclear charge polarization as a function of the pre-neutron mass. Results are compared to the Jeff libraries and the extrapolated points from light fragment measurement (P. Schillebeckx et al. [22])

\subsection{Isotopic and isomeric yields and their dependency on the kinetic energy}

Previous studies made by the collaboration show that under specific conditions, isomeric ratios (in that case $\mu$ s isomeric states) are dependent on fission fragment kinetic energy [15]. Measurements are obtained via a coincidence between an ionization chamber and the array of $\gamma$-rays detector. By using a $\gamma$ de-excitation code such as FIFRELIN, the angular momentum distribution of the fission product can be assessed. Recently the collaboration has developed similar measurements with the evolution of isotopic yields as a function of fission fragment kinetic energy. These results on the mass 139 are presented on Fig. 4 (left) in comparison to the FIFRELIN calculations. It represents a test for the models allowing the validation of the neutron and gamma cascade. Moreover we can extract the odd-even effect and neutron multiplicity as a function of excitation energy of nuclei (see Fig. 4 right).

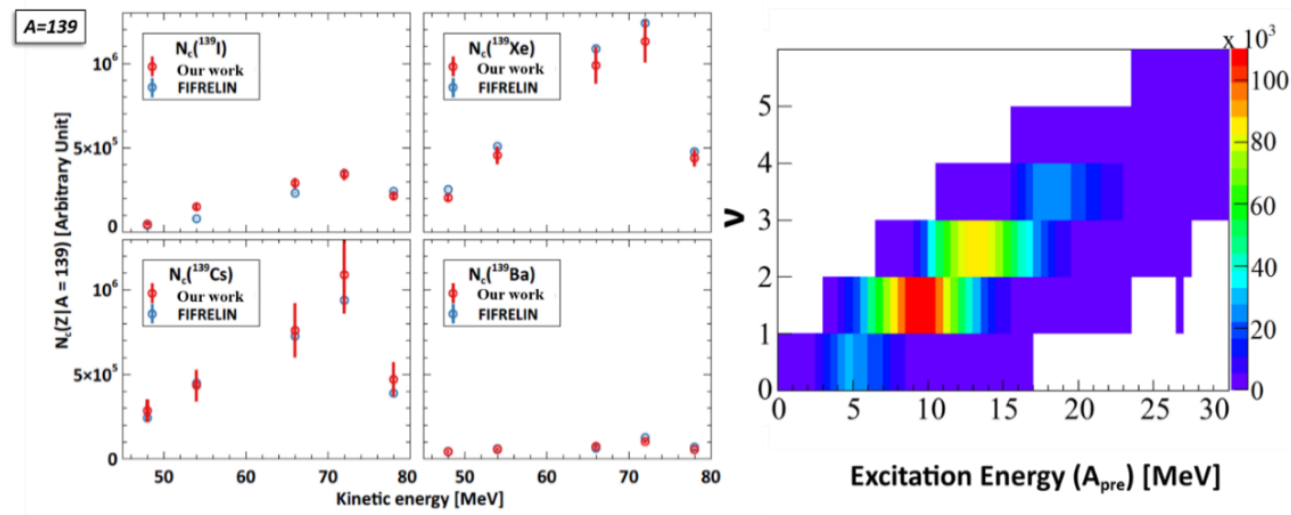


Fig. 4: (left) Kinetic energy dependence of cumulative isotopic yields for the mass 139 and the comparison to the FIFRELIN calculations. (Right) neutron multiplicity as a function of excitation energy deduced from FIFRELIN calculations.

\subsection{Nanosecond converted Isomeric Ratio}

Indirect measurement of nanosecond isomeric ratios can be determined from the ionic charge distribution [16-18]. The method consists in the deconvolution of the ionic charge distribution per isotope obtained by gamma spectrometry. The converted isomeric ratio (CIR) is defined as the converted isomer population over the total ionic population (converted and unconverted). According to the statistical models form H. Betz [19-20], we assume that the ionic charge distribution associated to the unconverted population follows a Gaussian distribution due to the charge equilibrium in the cover of the target (here Ni foils). A deviation from this Gaussian distribution indicates a charge changing due to the conversion from ps and ns isomers (see Fig. 5).CIR measurements are compared to FIFRELIN calculations (Fig. 6). We note a good tendency even if some differences have to be explored in details given the assumption used of the analysis or the models considered for the decay cascade calculations. It is interesting to note that this observable is not dedicated to one isomer but for all transitions at low energy. For certain nuclei, many isomers and bands are taking into account at the limit of the knowledge of the nuclear structure. Then it corresponds to integral measurements used to test the overall decay cascade.

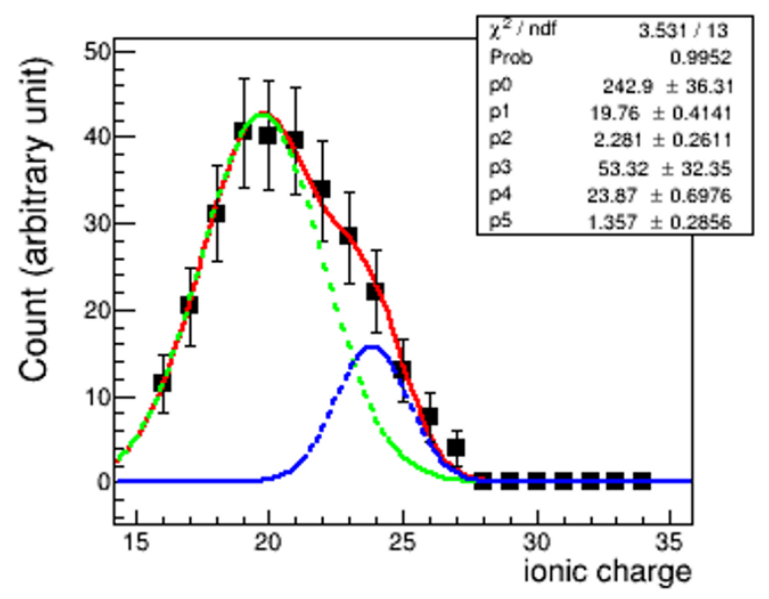

Fig. 5: Ionic charge distribution de-convoluted using the Gaussian assumption for the unconverted state according to the Betz model [19-20]. The blue curve corresponds to the ns-isomer contribution. 


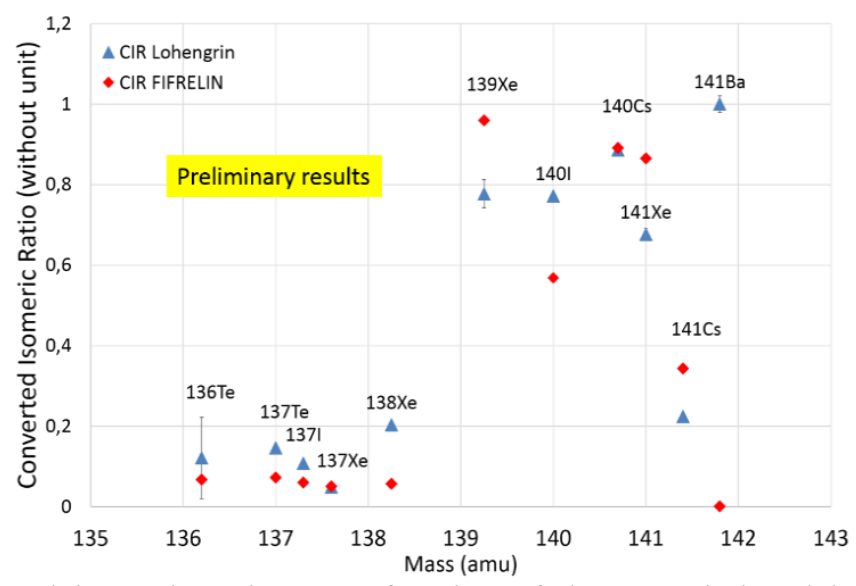

Fig. 6: Converted isomeric ratios as a function of the mass induced by the fission of ${ }^{241} \mathrm{Pu}\left(\mathrm{n}_{\mathrm{th}}, \mathrm{f}\right)$ in comparison to FIFRELIN calculations [18].

\section{Conclusion and perspectives}

A general status of the experimental program using the gamma spectrometry with the LOHENGRIN mass spectrometer at the ILL has been presented. Classical measurements of fission yields are now expanded with their kinetic energy dependency in order to test the limit of fission models. New measurement and validated models are central in order to progress in the evaluation project. Recent results for thermal neutron induced fission of ${ }^{241} \mathrm{Pu}$ have been presented. The gamma spectrometry method, in particular for the Converted Isomeric Ratios, considers the nuclear structure as the basic data allowing the study of a large timescale of isomeric states as probe of the fission process. In this topic, the development of the FIPPS project [21] at ILL is dedicated to the fission product spectroscopy and the fission process studies.

\section{Acknowledgements}

This work was supported by CEA, IN2P3 and "le défi NEEDS". The authors are grateful for the support of the ILL and all the staff involved from CEA Cadarache and LPSC.

\section{References}

1. O. Serot et al., Nuclear Data Sheets 119, 320-323 (2014)

2. F. Martin et al., Nucl. Data Sheets 119, 328 (2014)

3. S. Julien-Laferrière, A. Chebboubi, G. Kessedjian, O. Serot, EPJ N - Nuclear Sciences $\&$ Technologies, EDP Sciences, 2018, 4, pp. 25

4. N. Terranova, O. Serot, P. Archier, C. de Saint Jean and M. Sumini, Annals of Nuclear Energy 109, 469-489 (2017)

5. B. Voirin, G. Kessedjian, A. Chebboubi, O. Sérot, S. Julien-Laferriere et al., EPJ Nuclear Sci. Technol. 4, 26 (2018) 
6. H.R. Faust, P. Geltenbort, F. Gönnenwein and A. Oed, ILL Internal Scientific Report 81FA45S (1981)

7. G. Fioni, H. R. Faust, M. Gross, M. Hesse, P. Armbruster, F. Gönnenwein and G. Müzenberg, Nucl. Instrum. Meth. A 332, 175-180 (1993)

8. O. Litaize and O. Serot, Phys. Rev. C 82, 054616 (2010)

9. K. H. Schmidt and B. Jurado, Phys. Rev. C 83, 014607 (2011).

10. H. Faust et al., J. Korean Phys. Soc. 59, 879 (2011).

11. D. Regnier, $\mathrm{PhD}$ thesis, Grenoble University (2013)

12. J. P. Bocquet et al., Physics and chemistry of fission, IAEA, Proceedings of symposium, Jülich 1979.

13. F. Gönnenwein, I. Tsekhanovich, V. Rubchenya, International Journal of Modern Physics E, Vol. 16, No. 2 (2007) 410-424

14. H. Naik et al. Systematics of fragment angular momentum in low-energy fission of actinides. Nucl. Phys. A, $587: 273-290,1995$.

15. A. Chebboubi, G. Kessedjian, O. Litaize, O. Serot, H. Faust et al. Kinetic energy dependence of fission fragment isomeric ratios for spherical nuclei 132Sn, Phys. Lett. B, 2017, 775, pp.190-195.

16. T. Rzaca-Urban, J. Genevey, T. Materna et al., Phys. Rev. C 80, 064317 (2009)

17. A. Chebboubi et al., EPJ Web of Conferences, 146, (2017) 04021.

18. L. Thombansen, Master 1 report, Phelma engineering school, Grenoble, supervision G. Kessedjian, LPSC, 2018

19. H. D. Betz, Rev. Mod. Phys., 44 :465-539, Jul 1972.

20. H. D. Betz. Heavy Ion Charge States. In S. Datz, editor, Applied Atomic Collision Physics, Volume 4 : Condensed Matter, volume 4, page 2, 1983.

21. C. Michelagnoli et al., EPJ Web of Conferences, 193, 04009 (2018)

22. P. Schillebeeckx et al., Nucl. Phys. A, 580, 15-32, 1994. 